\title{
Forecasting of ionospheric characteristics during quiet and disturbed conditions
}

\author{
Iwona Stanisławska and Zbigniew Zbyszyński \\ Space Research Centre, Polish Academy of Sciences, Warsaw, Poland
}

\begin{abstract}
An autocovariance forecasting procedure for single location ionospheric characteristics is presented. Its accuracy is illustrated as a function of the amount of time extrapolation for selected European stations under quiet and disturbed conditions.
\end{abstract}

Key words forecasting of ionospheric characteristics

\section{Introduction}

Day-to-day and hour-to-hour ionospheric variations are generally irregular. Irregular ionospheric variations are variations that cannot be predicted by any linear prediction method. They are caused by irregular changes in amplitudes of ionospheric parameters as well as their time spread. In order to predict them much interest and efforts have been dedicated in the past. Various non-linear techniques have been proposed. The latest publications deal with a statistical approach (Muhtaov and Kutiev, 1999), or with a modern neural network technique (Wintoft and Cander, 1999, 2000; Tulunay et al., 2000). This paper presents a continuation of this work (Stanisławska and Zbyszyński, 2001) in the application of the autocovariance prediction method for ionospheric purposes.

Mailing address: Dr. Iwona Stanisławska, Space Research Centre PAS, 00-716 Warsaw, Bartycka 18a, Poland; e-mail: stanis@cbk.waw.pl

\section{The autocovariance prediction method application}

The autocovariance prediction method was originally elaborated at the Space Research Centre for prediction of irregular variations in Earth rotation (Kosek, 1993, 1997). In this method the first prediction point outside the data time interval in the future and in the past is computed and added at the beginning or at the end of data, respectively, so the next prediction point can be computed. The difference between the prediction and data at a particular time in the future computed at different starting prediction epochs reveals unpredictable or irregular variations of the considered ionospheric parameter. Along this line the analysis of potential application of this method in the ionosphere was presented in Stanisławska (1994). The forecasting capabilities of the method for $f_{0} F_{2}$ parameter were shown in Stanisławska and Zbyszyński (2001). This paper presents a similar analysis for other ionospheric characteristics as well as the forecast dependence on the time range considered.

One of the advantages of the autocovariance method is that it does not require any additional parameters, which describe solar and/or geophysical conditions. It also means that any 
additional uncertainty connected with the need to use a prediction of these is avoided. The only information needed is a long enough period of observation. Another advantage is that it can be used for forecasting without any knowledge of the morphological and physical processes in a medium, such as the ionosphere.

\section{Results and conclusions}

The present study investigates the $f_{0} F_{2}, f_{0} F_{1}$, $f_{0} E$, and $M(3000) F_{2}$ parameters. Data have been taken from RAL-CD-ROM, prepared by the Rutherford Appleton Laboratory, United Kingdom, for European Cooperation in the Field of Scientific and Technical Research (COST) Action 251 (Hanbaba, 1999) and from the Ionospheric Despatch Centre in Europe (Stanislawska et al., 1999) (http://www.cbk.waw.pl/rwc/idce.html), that is a COST 251 initiative. A list of relevant stations is presented in table I.

In this method the prediction estimation is computed as a function of an observed variable (different ionospheric characteristic) from several ionospheric stations only. The sampling interval of ionospheric characteristics, in our case, is $1 \mathrm{~h}$. In this paper, 1-, 2-, 4-, 8-, 12-, 24- and 48-h-ahead forecast have been obtained. Particular purposes of this application are to consider the needs of the forecast obtaining for instantaneous situation by operational service of the ionospheric situation. To deal with a real situation any specific method for gaps filling has been used. Any data gaps were replaced by 7-days-smoothed average only. Also the requirements of the time period of available data were limited. Data from separate periods within September - November 1998 were used. The number of data used in the computation for the presented statistics is shown in table II.

For the calculations of the short time forecast (up to $12 \mathrm{~h}$ ahead) 73 past values ( 3 days) hour by hour, have been taken, while for a longer time forecast (24-48 h ahead) 28 values from the previous 28 days, for each hour separately, have been taken. To satisfy the requirements of the method, as input data we used deviations of the measurements from the 7-days-smoothed

Table I. Stations and their geographical coordinates.

\begin{tabular}{cccc}
\hline \hline Location & Station & Latitude, ${ }^{\circ} \mathrm{N}$ & Longitude, ${ }^{\circ} \mathrm{E}$ \\
\hline Tortosa & EB040 & 40.8 & 0.5 \\
Rome & RO041 & 41.9 & 12.5 \\
Juliusruh & JR055 & 54.6 & 13.4 \\
Sofia & SQ143 & 42.7 & 23.4 \\
Warsaw & MZ152 & 52.2 & 21.2 \\
Uppsala & UP158 & 59.8 & 17.6 \\
Lycksele & LY164 & 64.6 & 18.8 \\
Kiruna & KI167 & 67.8 & 20.4 \\
\hline
\end{tabular}

Table II. Number of considered data points.

\begin{tabular}{cccc}
\hline \hline & Disturbed & Quiet & Total \\
\hline$f_{0} E$ & 1900 & 3900 & 5800 \\
$f_{0} F_{1}$ & 500 & 900 & 1400 \\
$f_{0} F_{2}$ & 6100 & 9200 & 15300 \\
$M(3000) F_{2}$ & 6100 & 8500 & 14600 \\
\hline
\end{tabular}



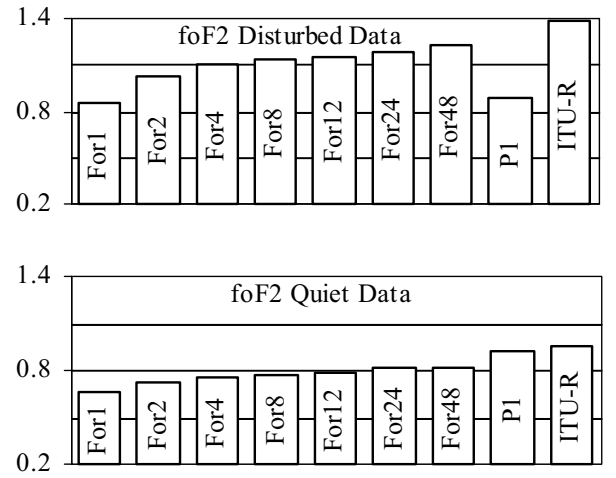

(1)
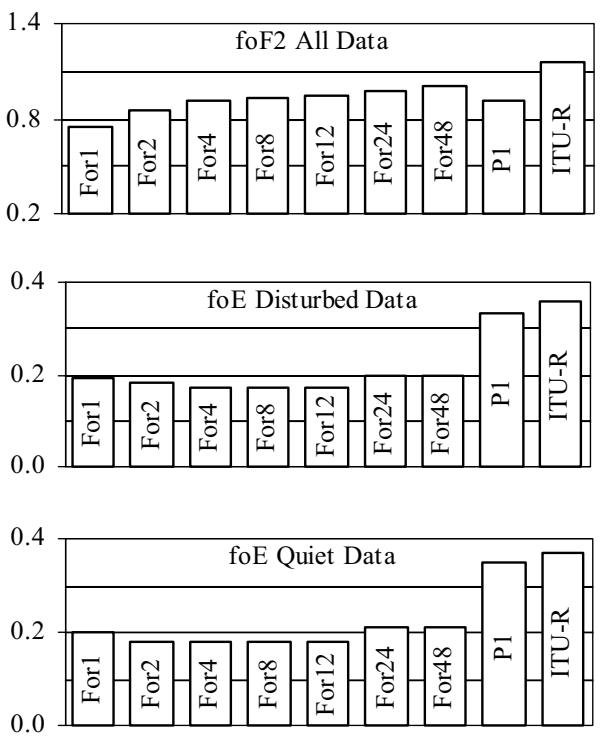

(3)

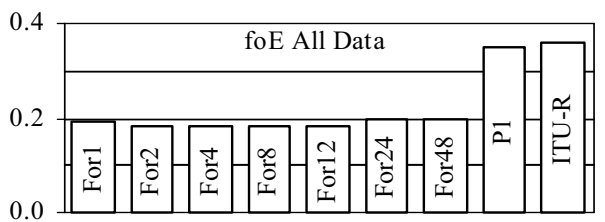

(2)
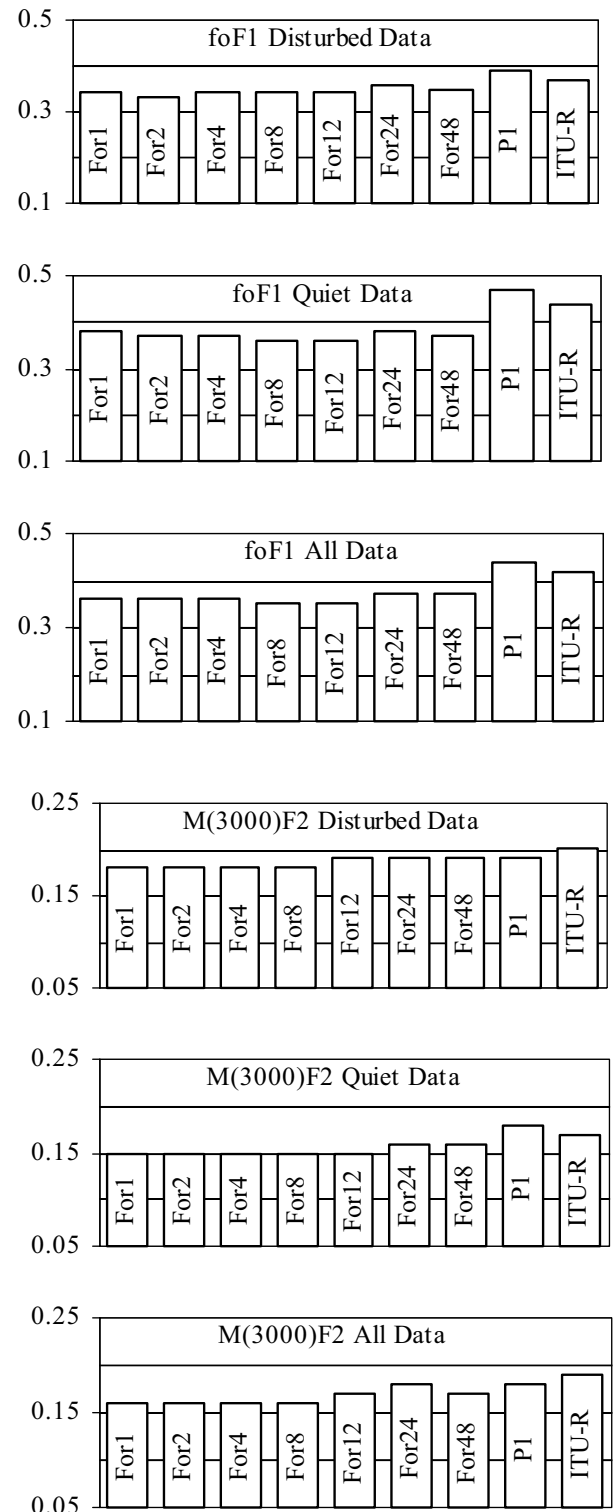

Fig. 1. Bar chart created for RMS errors for $f_{0} F_{2}$ characteristic, for disturbed data (upper panel), quiet data (middle panel), and for all data together (lower panel). Autocovariance forecasting method: For1, $1 \mathrm{~h}$ ahead For2, $2 \mathrm{~h}$ ahead, etc. Persistence: P1.

Fig. 2. The same as fig. 1 for $f_{0} F_{1}$ characteristic.

Fig. 3. The same as fig. 1 for $f_{0} E$ characteristic.

Fig. 4. The same as fig. 1 for $M(3000) F_{2}$ characteristic. 
average value calculated for each hour separately. An additional correction factor for 1-hahead forecast is introduced in the following manner: for the forecast at time $t$, parameters at times $t-1$ and $t-2$ are calculated. If the values in $t-1$ and $t-2$ differ from the measurements by more than $30 \%$, the current calculated forecast is changed exactly by the last value of the gradient. The forecast was analysed for the disturbed and quiet periods separately. For the distinction between quiet and disturbed conditions, the catalogue of disturbances (Kouris et al., 1998) observed at selected ionospheric stations was used. The catalogue is available at the Ionospheric Despatch Centre in Europe.

The prediction error is presented as an RMS error and percentage deviation of the prediction against the measurements. For comparison with the forecast results using the ITU-R-predicted median value and the measurement from the previous hours (persistence) have also been given.

Figures 1 to 4 show the bar charts of the RMS error for four considered characteristics for quiet and disturbed conditions separately, as well as for all data together. The method has been used
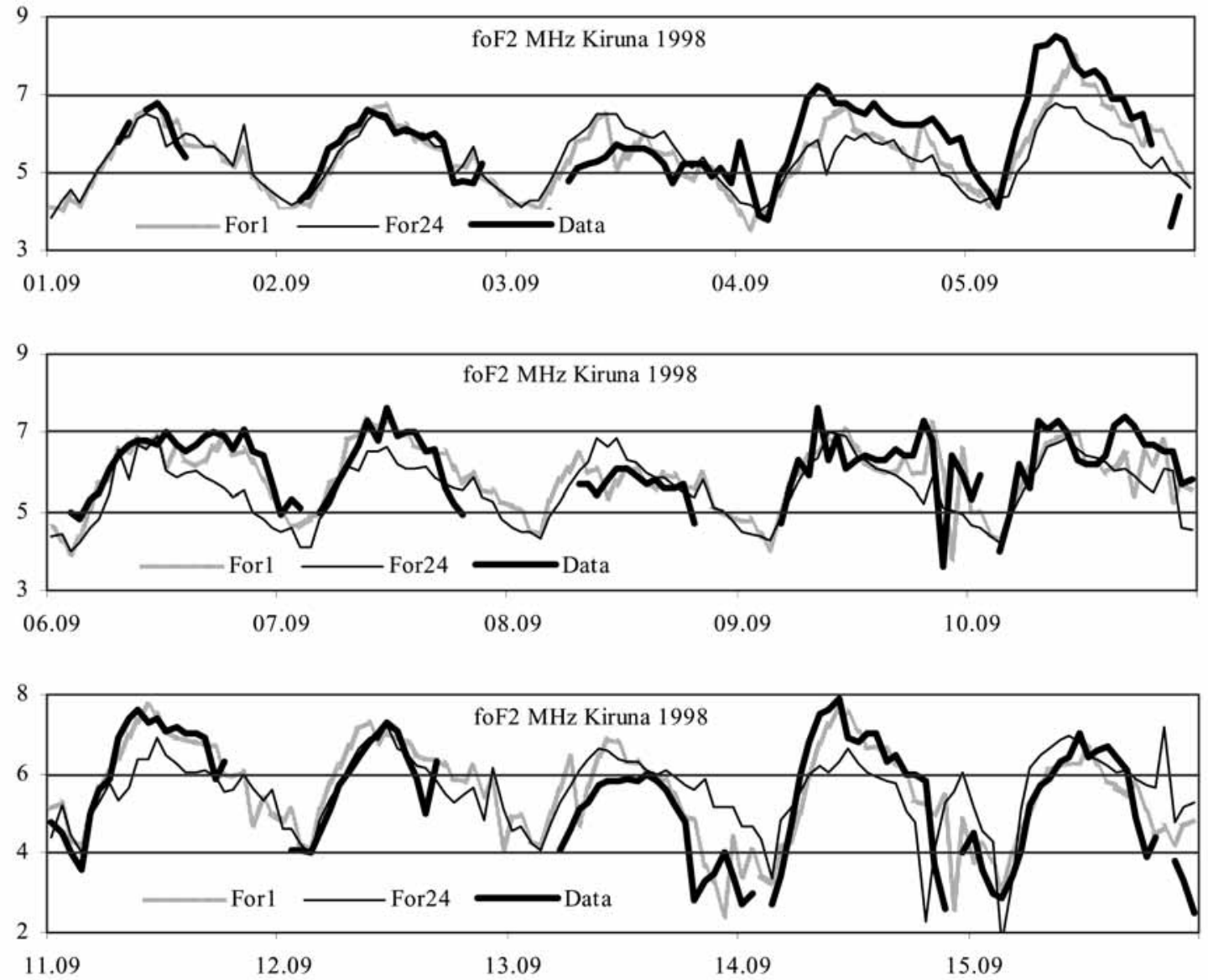

Fig. 5. 1- and 24-h-ahead forecast, and measurements for $f_{0} F_{2}$ characteristic for three 5-day-periods in September 1998 at Kiruna station. 

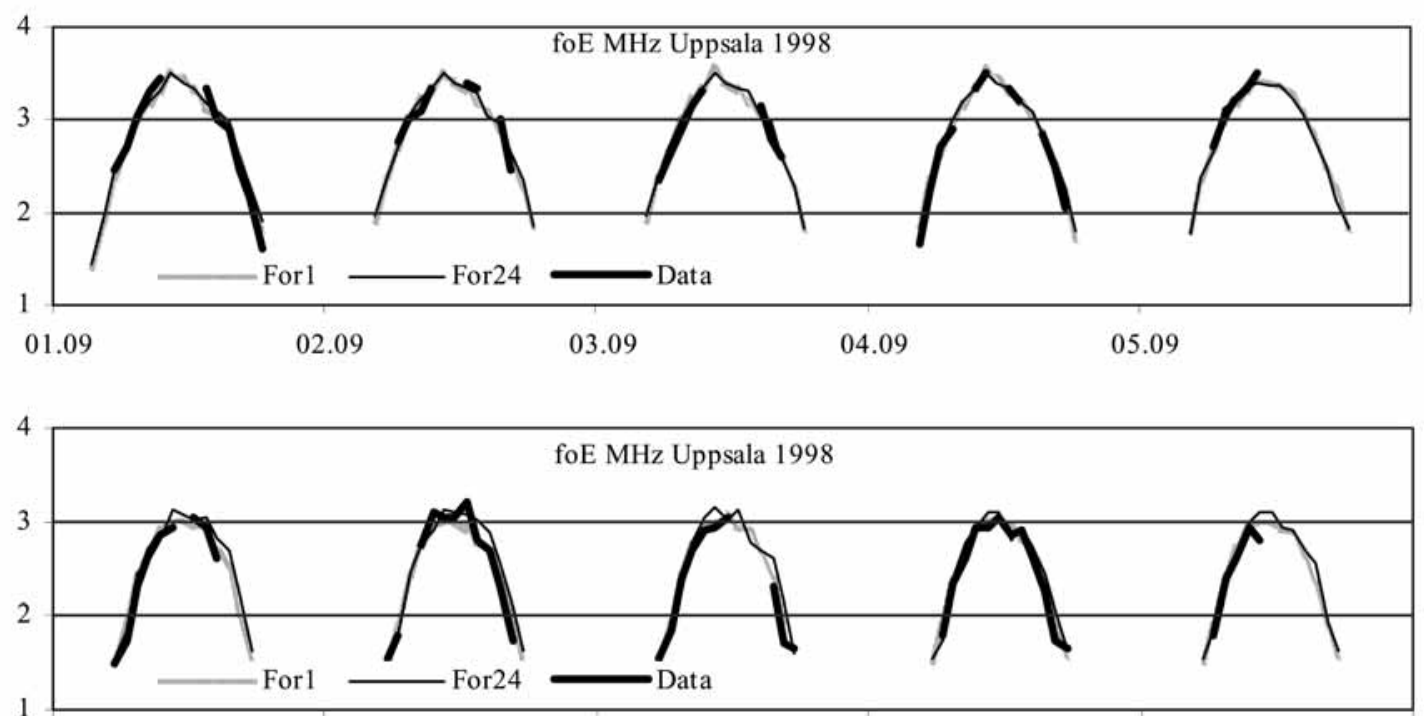
06.09
07.09
08.09
09.09
10.09

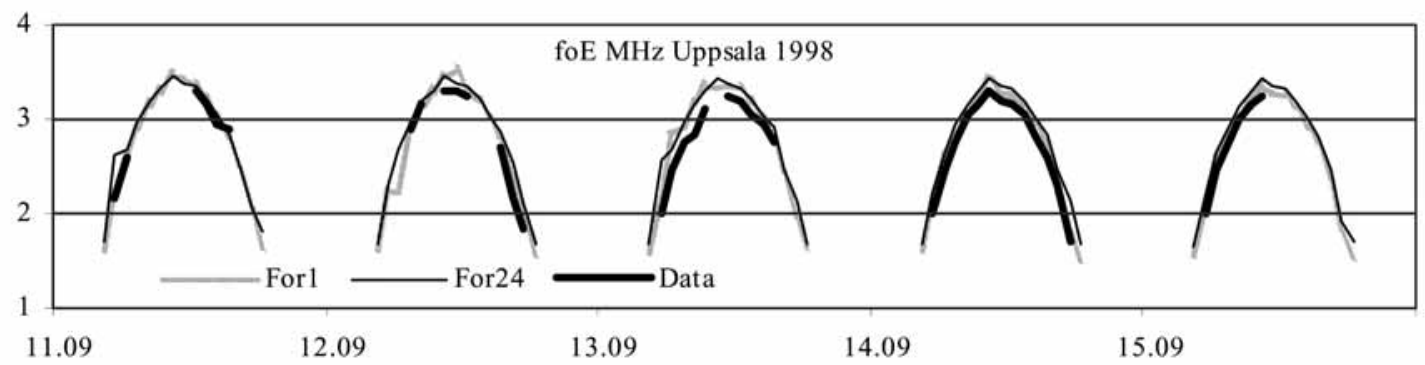

Fig. 6. The same as fig. 5 for $f_{0} E$ characteristic at Uppsala station.

for 1-, 2-h ahead, etc. Samples of 1-, and 24-hahead forecast for $f_{0} F_{2}, f_{0} E$ and $M(3000) F_{2}$ characteristics for the most quiet, incidentally chosen moderate disturbed and the most disturbed periods are shown in figs. 5 to 7 . Five day periods in October and September 1998 observations, 1- and 24-h ahead, are presented. Incidentally chosen, but representative for the numerical experiments performed, stations from mid- and high latitudes are presented. Only the plot for $f_{0} F_{1}$ characteristic (fig. 8) is presented for a 5-day-period in September 1998 for a mixed quiet and negatively disturbed period for the mid-latitude Warsaw station only.
For all the considered data the forecast shows much better results than medians and persistence. Except for the most disturbed data for $f_{0} F_{2}$ characteristic, when the persistence is better than the forecast 2-, 4-, etc. hours ahead. But obviously, while considering the forecast for longer than 1-h-ahead period, the persistence does not exist. It ought to be mentioned that ITU-R prediction is never available with such accuracy, as used in this paper, because of the actual solar activity parameters used (not prediction). For $M(3000) F_{2}$ characteristic, increasing the time range of the forecast also increases the errors. This increase is not so 

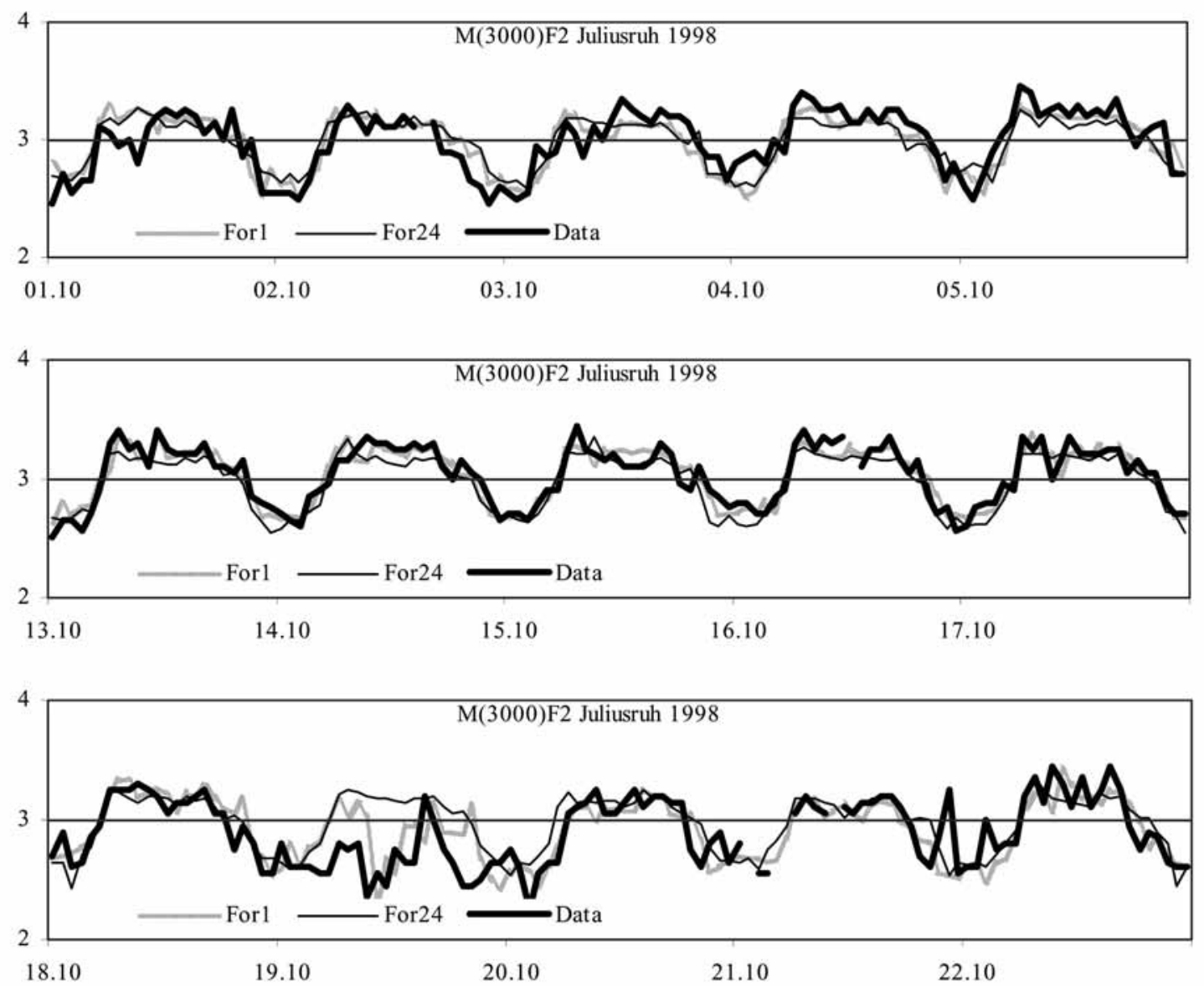

Fig. 7. The same as fig. 5 for $M(3000) F_{2}$ characteristic for three 5-day-periods in October 1998 at Juliusruh station.

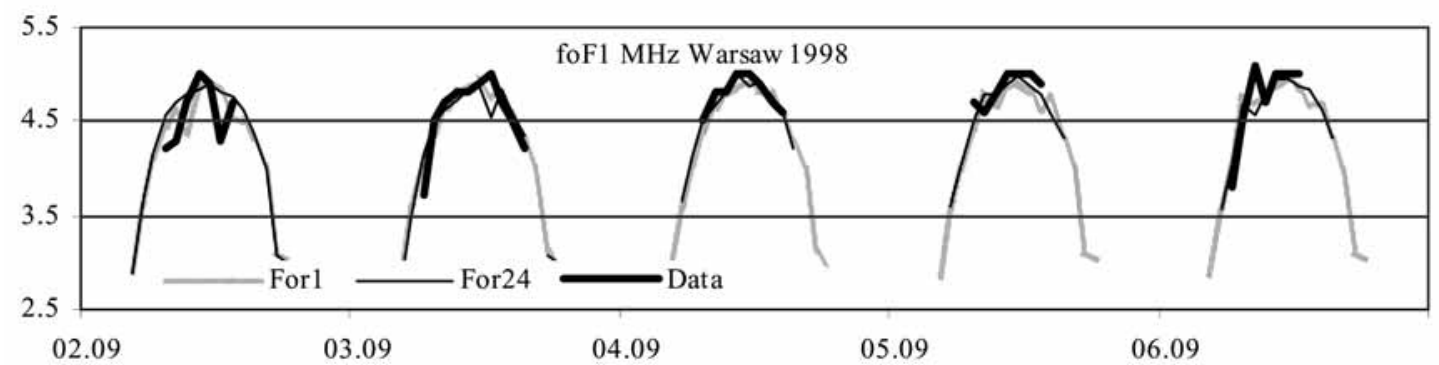

Fig. 8. The same as fig. 5 for $f_{0} F_{1}$ characteristic for one 5-day-period in September 1998 at Warsaw station. 
pronounced as for $f_{0} F_{2}$. For $f_{0} F_{1}$ and $f_{0} E$ characteristics the situation is quite different. The impact of the data from sunrise and sunset hours, particularly for $f_{0} F_{1}$ data, and night hours for $f_{0} E$, enlarge the errors, because the forecast is sometimes given for non-existing $F_{1}$ layer, and vice versa. Generally, the quiet situation, which is represented by much more smoothed data than for the disturbances, is predicted with higher accuracy. During the disturbance lasting several hours, the method might generate some rapid fluctuations. When the method gives too high, or too low values, the correction by gradients improves the forecast, but only for the second, and higher measured values hours. So the forecast curve for later hours is much smoother and closer to observations. However, for individual disturbances the errors might still be substantial. This effect can be avoided using data with higher resolution sampling, as 15 , or 5 min. In such a case 1-h-ahead forecast will follow the observations with higher accuracy after half an hour, or $10 \mathrm{~min}$, respectively.

Generally, the autocovariance method shows the correctness of this approach for any ionospheric characteristics. The autocovariance method of ionospheric characteristics forecasting provides an acceptable accuracy. This might also be the crucial point for predicting the electron concentration height profiles. Its quite reliable results for quiet, as well as for disturbed conditions allow us to conclude that this method can be used in operational services of ionospheric situation, as used to update the limited-area ionospheric forecast at the Regional Warning Centre Warsaw of the International Space Environment Service.

\section{Acknowledgements}

This research was partly supported by Polish Committee of Scientific Research grant 2 P03C 00617.

\section{REFERENCES}

HANBABA, R. (1999): COST 251 Final Report, SRC Print. Off., Warsaw.

KoseK, W. (1993): The autocovariance prediction of the Earth rotation parameters, in 7th International Symposium "Geodesy and Physics of the Earth», IAG Symposium No. 112, Potsdam, Germany, October 5-10, 1992, edited by H. Montag and Ch. ReIGBer (Springer Verlag), 443-446.

KosEK, W. (1997): Autocovariance prediction method of short period Earth rotation parameters, Artif. Earth Satell., 32 (2), 75-85

KouRIS, S.S., D.N. Fotiadis and T.D. Xenos (1998): On the day-to-day variation on $f_{0} F_{2}$ and $M(3000) F_{2}, A d v$. Space Res., 22 (6), 873-876.

MUHTAROV, P. and I. KUTIEV (1999): Autocorrelation method for temporal interpolation and short-term prediction of ionospheric data, Radio Sci., 34 (2), 459-464.

STANISKAWSKA, I. (1994): A single-station prediction model as a contribution to instantaneous mapping, Ann. Geofis., 37 (2), 153-157.

STANISKAWSKA, I. and Z. ZBYSZYNŚSKI (2001): Forecasting of the ionospheric quiet and disturbed $f_{0} F_{2}$ values at single location, Radio Sci., 36 (5), 1065-1071.

StANISLAWSKA, I., T.L. GULYAEVA and R. HANBABA (1999): Ionospheric Despatch Centre in Europe, Phys. Chem. Earth (C), 24 (4), 355-357.

Tulunay, E., C. OzKaPtan and Y. Tulunay (2000): Temporal and spatial forecasting of the $f_{0} F_{2}$ values up to twenty four hours in advance, Phys. Chem. Earth $(C)$, 25 (4), 281-285.

WINTOFT, P. and LJ.R. CANDER (1999): Short-term prediction of $f_{0} F_{2}$ using time-delay neural network, Phys. Chem. Earth $(C), 24$ (4), 342-347.

WINTOFT, P. and LJ.R. CANDER (2000): Ionospheric $f_{0} F_{2}$ storm forecasting using neural networks, Phys. Chem. Earth (C), 25 (4), 267-273. 